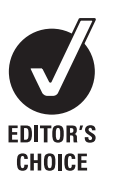

CHOICE

\title{
Extraocular surgery for implantation of an active subretinal visual prosthesis with external connections: feasibility and outcome in seven patients
}

\author{
D Besch, ${ }^{1}$ H Sachs, ${ }^{2}$ P Szurman, ${ }^{1}$ D Gülicher, ${ }^{3}$ R Wilke, ${ }^{1}$ S Reinert, ${ }^{3}$ E Zrenner, ${ }^{1}$ \\ K U Bartz-Schmidt, ${ }^{1}$ F Gekeler ${ }^{1}$
}

${ }^{1}$ Centre for Ophthalmology, University of Tübingen,

Tübingen, Germany:

${ }^{2}$ Department of Ophthalmology, University of Regensburg,

Regensburg, Germany;

${ }^{3}$ Department of Oral and

Maxillofacial Surgery, University

of Tübingen, Tübingen, Germany

Correspondence to:

Dr F Gekeler, Centre for

Ophthalmology, University of

Tübingen, Schleichstraße 12-

16, 72076 Tübingen, Germany: gekeler@uni-tuebingen.de

Accepted 15 July 2008

Published Online First

28 July 2008

\begin{abstract}
Background: Due to low energy levels in microphotodiode-based subretinal visual prostheses, an external power supply is mandatory. We report on the surgical feasibility and the functional outcome of the extraocular part of an approach to connect a subretinal prosthesis to an extracorporeal connector in the retro-auricular space via a trans-scleral, transchoroidal cable.
\end{abstract}

Methods: Seven volunteers with retinitis pigmentosa received an active subretinal implant; energy was supplied by gold wires on a trans-sclerally, transchoroidally implanted polyimide foil leading to the lateral orbital rim where it was fixated and connected to a silicone cable. The cable was implanted subperiostally beneath the temporal muscle using a trocar to the retroauricular space where it penetrated the skin for connection to a stimulator. To avoid subretinal movement of the implant, three tension relief points have been introduced.

Results: All implantations were performed as planned without complications, and no serious adverse events occurred in the postoperative period. Fixation of the implants was stable throughout the entire study duration of 4 weeks; permanent skin penetration proved to be uncomplicated. Motility was minimally restricted in downgaze and ab-/adduction. Explantation was uneventful.

Conclusion: The above-described procedure provides a method for stable fixation of a subretinal device with a trans-scleral, transchoroidal cable connection to an extracorporeal connector.

Retinal implants elicit visual perceptions by electrical stimulation of retinal cells. ${ }^{2}{ }^{2}$ They are primarily designed for patients suffering from degenerative retinal disease such as retinitis pigmentosa (RP) where outer retinal cells deteriorate while inner retinal cells stay intact a longer time $e^{34}$ and can be used to transmit electronically generated signals to the brain. Our group has proposed a device based on microphotodiodes which are implanted into the subretinal space and deliver electrical pulses by transforming light energy into electrical energy. ${ }^{5-8}$ However, this energy is insufficient to excite retinal cells even under bright light. ${ }^{9-}$ ${ }^{12}$ Therefore, additional energy has to be supplied to such implants, for example via a cable connection. To this purpose, our group has developed a compound visual prosthesis with connections from a subretinal microphotodiode array (MPDA) to an extracorporeal connector in the retro-auricular space via a transchoroidally, trans-sclerally implanted polyimide foil. A transcutaneous cable passage to an extracorporeal connector can certainly only be a transitional step, but for the limited duration of our pilot study (implantation was granted for 4 weeks) a direct cable connection offered us several important advantages. More importantly, this allowed us to directly assess parameters such as charge transfer and impedance of individual subretinal electrodes, which are paramount for the understanding of the interaction of electrodes and neural tissue. To our knowledge, these data have not been gathered before from subretinal electrical stimulation in long-term experiments. In addition to that, several control parameters of MPDA function were directly measurable and adjustable in these first experiments which proved to be crucial for optimisation of these functions.

For implantation of a prosthesis with a transchoroidal, trans-scleral connection, our group has established a surgical approach in animal tests. ${ }^{6713-18}$ The experience gained from these successful experiments led to optimisation of the technique that would be suitable for humans. Of high importance were tension relief points along the implant's extraocular course to prevent transduction of movements of the extraocular to the subretinal parts which had resulted in frequent retinal tears and subsequent detachments in research animals.

We report here on the success of the extraocular surgical approach to connect the subretinal device via a transchoroidal and transcutaneous cable to an extracorporeal connector in seven human volunteers suffering from RP.

\section{MATERIAL AND METHODS \\ Patients}

Seven male patients with end-stage RP were included. Remaining vision was required to be "of no use in daily life" by the local ethics committee. Diagnosis was established by family history, electroretinography, fluorescein angiography, optical coherence tomography, slit-lamp examination and funduscopy. In all subjects, electrical excitability was ensured by prior transcorneal electrical stimulation with DTL electrodes. ${ }^{19}$ All experiments were undertaken with the understanding and written consent of each subject 

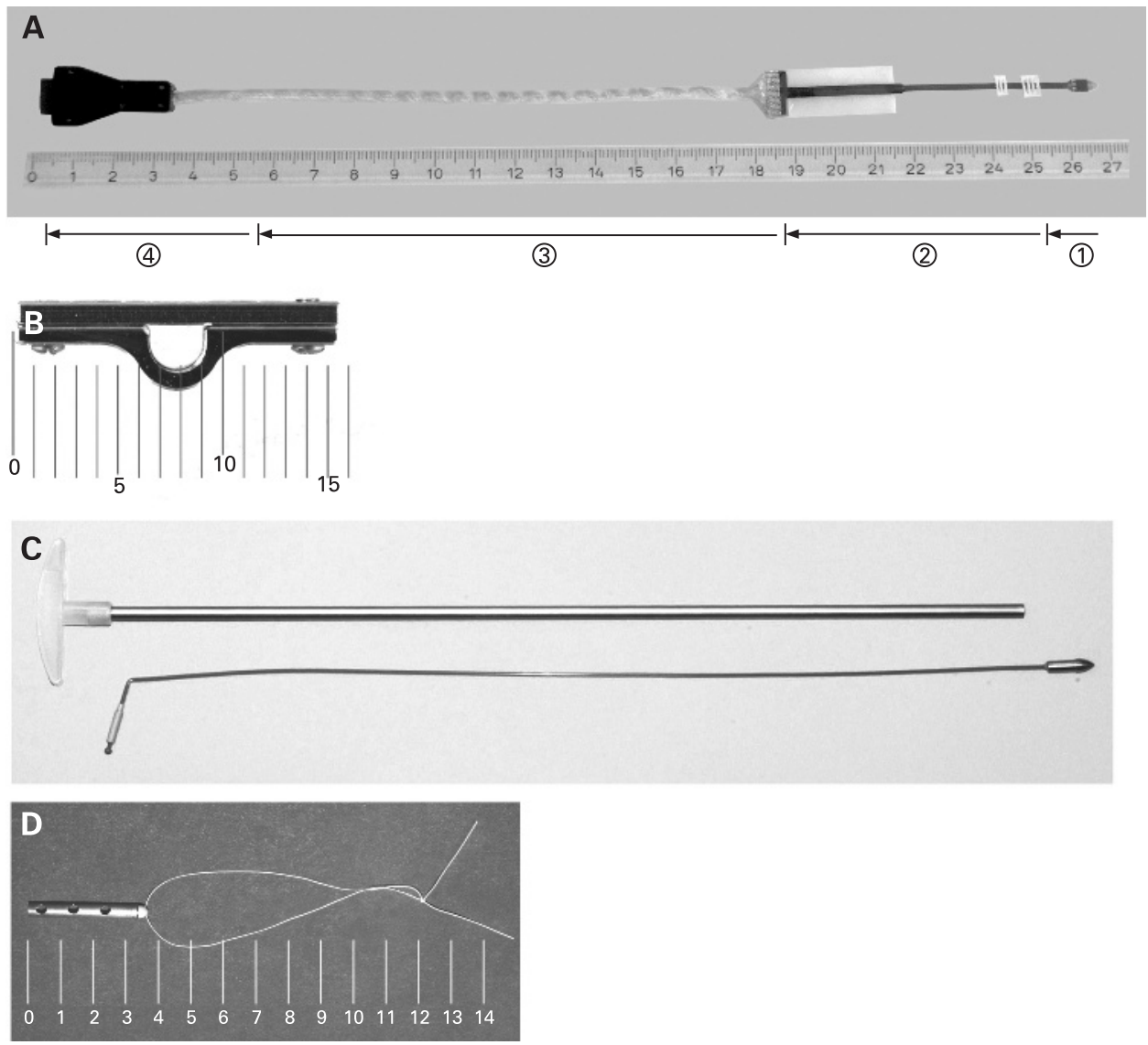

Figure 1 Photography of the active subretinal implant with external connections and surgical adjuvants. (A) The implant consists of four parts: (1) The subretinal part (polyimide foil strip, $20 \mu \mathrm{m}$ thick, $3.2 \mathrm{~mm}$ wide, approximately $22 \mathrm{~mm}$ long) with: a $4 \times 4$ array of titanium-nitride electrodes (diameter $50 \mu \mathrm{m}$, spacing $280 \mu \mathrm{m}$ ) and a microphotodiode array with 1550 photodiodes and electrodes (electrode diameter $70 \mu \mathrm{m}$, spacing $70 \mu \mathrm{m}$, thickness $70 \mu \mathrm{m}$ ) which lies parafoveally. (2) The extraocular part (polyimide foil) carrying 22 golden connection lanes to the external connection and the reference electrode. At a length of 12 and $26 \mathrm{~mm}$ from the tip, two polytetrafluoroethylene pads are mounted for fixation on the sclera. At approximately $55 \mathrm{~mm}$ from the tip, a silicone pad is glued to the polyimide foil for suture fixation to the lateral orbital rim. (3) The subdermal part (silicone cable, diameter $3 \mathrm{~mm}$ ) leads the connections on the polyimide foil to the external part by 22 spiralled gold flexes. The silicone cable perforates the retro-auricular skin. (4) The external part consists of the silicone cable which connects via a plug to an external generator for control signals, energy and stimuli. (B) Clamp for fixation of the silicone cable in the retro-auricular scull region (scale in $\mathrm{mm}$ ). (C) Aluminium trocar (diameter $6 \mathrm{~mm}$, length approximately $40 \mathrm{~cm}$ ) for subperiostal placement of the implant. (D) Improved cartridge for trans-septal passage of the implant used in the last patients. The tip of the implant carrying the photodiode array and the electrodes for direct stimulation are placed inside the cartridge, which is then carefully pulled from the orbital rim through the septum to the upper temporal quadrant of the eye for subretinal implantation (dimensions in mm).

respecting the Code of Ethics of the World Medical Association (Declaration of Helsinki) and in accordance with the European Communities Council Directive of 24 November 1986 (86/609/ EEC). The study was approved by the local university's ethics committee which granted permission for a study period of 4 weeks.

\section{Subretinal prosthesis}

The prosthesis consisted of three parts (figs 1A, 2).

1. The subretinal part, with (A) the MPDA with 1550 photodiodes and titanium-nitride electrodes (this is driven by light falling onto it through the optics of the eye and powered by external energy transferred via golden connection lanes on the polyimide foil strip) and (B) 16 titaniumnitride electrodes in a $4 \times 4$ array for direct, light-independent electrical stimulation; each electrode is connected to an outside stimulus generator via golden connection lanes on the trans-sclerally, transchoroidally implanted polyimide foil strip.

2. The extraocular part. After choroidal and scleral penetration, the foil strip is flipped to lie episclerally. It then leads under the lid to the lateral orbital rim. For fixation, a patch of polytetrafluoroethylene glued to the foil is sutured onto the sclera. At the end of the foil strip, a silicone patch is fixated by sutures in two holes through the lateral orbital rim.

3. The subdermal part. The foil strip connects in the temporal fossa to a silicone cable with spirally twisted, isolated wires, which leads subperiostally beneath the temporal muscle to the retro-auricular space where it penetrates the skin and ends in a plug. There, the device is fixed to the bone by a stainless steel clamp (figs 1B, 2).

\section{Surgical technique}

\section{Implantation}

The implantation procedure was carried out sequentially in the following steps (fig 3).

After subcutaneous injection of mepivacaine/epinephrine in the retro-auricular space the skin was incised in arcuate form at a length of $5 \mathrm{~cm}$. At the height of the external acoustic meatus approximately $2 \mathrm{~cm}$ by $2 \mathrm{~cm}$ of bone were exposed by use of a raspatory. Two holes were drilled and the base plate of the 


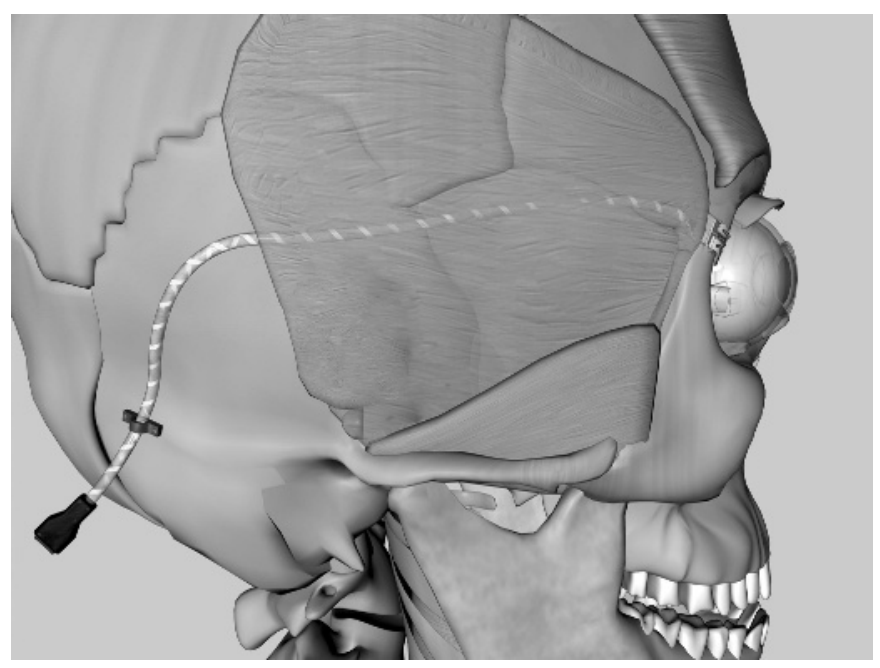

Figure 2 Schematic drawing of the course of the subretinal prosthesis under the temporalis muscle as implanted in seven human volunteers.

clamp (fig 1B) fixed with osteosynthesis screws. Surgery was then continued in the periorbital region with a prolonged brow incision to expose the lateral orbital rim. Two holes were drilled $2 \mathrm{~mm}$ superior and inferior to the sutura zygomatico-frontalis, and Vicryl 3-0 threads were inserted. Preparation proceeded laterally into the fossa temporalis elevating the temporalis muscle and the periosteum. Following that, a $360^{\circ}$ peritomy of the conjunctiva was performed at the limbus. From the subconjunctival space in the upper temporal quadrant, a tunnel was bluntly prepared through the septum to the brow incision and was kept patent by a small silicone tube. A specially made trocar (fig 1C) was advanced from the periorbital region subperiosteally until it reached the retro-auricular area. The implant could then be advanced inside in anterior direction and the trocar be removed leaving the implant in place. The tip of the implant was placed inside of the silicone tube which held the subconjunctival tunnel open. The tube protected the implant's tip when it was pulled through the tunnel to the subconjunctival space and placed aside securely during the following intraocular surgical procedure. In brief, the intraocular surgery comprised a parsplana-vitrectomy, creation of a subretinal bubble and transchoroidal penetration in the upper lateral quadrant of the eye. Then, the fixation pad was sutured onto the sclera and the retina reattached by perfluorocarbon liquids and filled with silicone oil for endotamponade.

Subjects received the following postoperative medication: dexamethasone $(250 \mathrm{mg}$ intravenously on the day of the surgery and thereafter; up to $100 \mathrm{mg}$ orally for 2 weeks), $500 \mathrm{mg}$ of ciprofloxacinhydrochloride orally twice daily for 10 days, dexamethasone/gentamycin eye-drops four times daily. The area of skin penetration in the retro-auricular space was treated daily with povidone iodine solution and covered by medical tape. The skin sutures at the orbital rim were treated for 1 week with gentamycin ointment.

\section{Explantation}

Skin wounds were reopened in the retro-auricular space and in the brow region to expose the implant. The silicone cable was dissected at the junction with the polyimide foil in the fossa temporalis. The attachment screws of the clamp in the retroauricular space were removed and the silicone cable pulled posteriorly; the basal plate of the clamp and the bone screws were removed. Intraocular parts of the implant were removed by loosening the scleral fixation pad, and the scleral access was reopened to pull out the intraocular parts. No intraocular procedure was required. All wounds were finally closed in layers. Silicone oil was removed approximately 3 months later, depending on the individual situation.

\section{RESULTS}

\section{Feasibility of extraocular surgery}

All prostheses could be safely implanted using the above described procedure. No intraoperative complications were encountered. Surgery was performed in all cases without damaging the prosthesis. Figure 3 shows the decisive surgical steps in one patient.

Due to the experience from surgical trials in animals and human cadavers, the surgical procedure provided no major difficulties. The procedure of subperiostal implantation from the retro-auricular space to the orbital rim using the trocar proved to be easily controllable in all cases. In general, the most challenging part was the protection of the implant during all steps of the surgery; touching the MPDA or the electrodes was not possible with any instrument, and even fine threads could easily tear the polyimide foil and lead to disruption of the gold wires (besides constituting a predetermined breaking point). An especially delicate part turned out to be pulling the polyimide foil with the subretinal parts through the opening from the lateral orbital rim under the lateral lid to the subconjunctival space: first, because the fairly large protecting silicone tube exerted considerable pressure onto the globe leading to a marked increase in intraocular pressure requiring fast manoeuvres; second, because the delicate subretinal parts had to be protected against mechanical injury by placement inside the silicone tube while pulling the tube through the iatrogenic access. To facilitate this and provide better protection for the globe and the implant, a metal cartridge has been used in the last three patients. The cartridge was smaller in diameter, and a thread could be attached to pull it through the iatrogenic access (fig 1D); it considerably reduced pressure increases on the globe and helped to protect the implant's tip. Coagulation by monopolar or bipolar diathermia was to be avoided in the proximity of the implant because high-frequency electrical fields could damage the MPDA which acted as an antenna. To protect the implant, a large spatula (touching the sclera to conduct any currents) was placed between it and the coagulating tip. During the following intraocular surgery, the implant tip was kept away inside the silicone/metal tube taped to the surgical drape.

The time for extraocular surgery was $1.5-2.5 \mathrm{~h}$; the whole surgery lasted 6-7 h.

\section{Surgical outcome}

During the first postoperative days moderate oedema and haematoma in the periorbital region were noticeable (fig 4). The subdermal passage to the retro-auricular space proved to be unproblematic in all cases, leading to only minimal swelling (fig 4) and almost no pain. Two patients complained about a mild jaw closure blockade noticed during chewing which had disappeared 1 week postoperatively. A possible cause is an irritation of the temporal muscle or some fibres of the facial nerve in this region of the cable passage. Conjunctival chemosis and injection were observed in all patients comparable with other intraocular procedures such as pars-plana-vitrectomy. After approximately 5 days, conjunctival chemosis, oedema and 

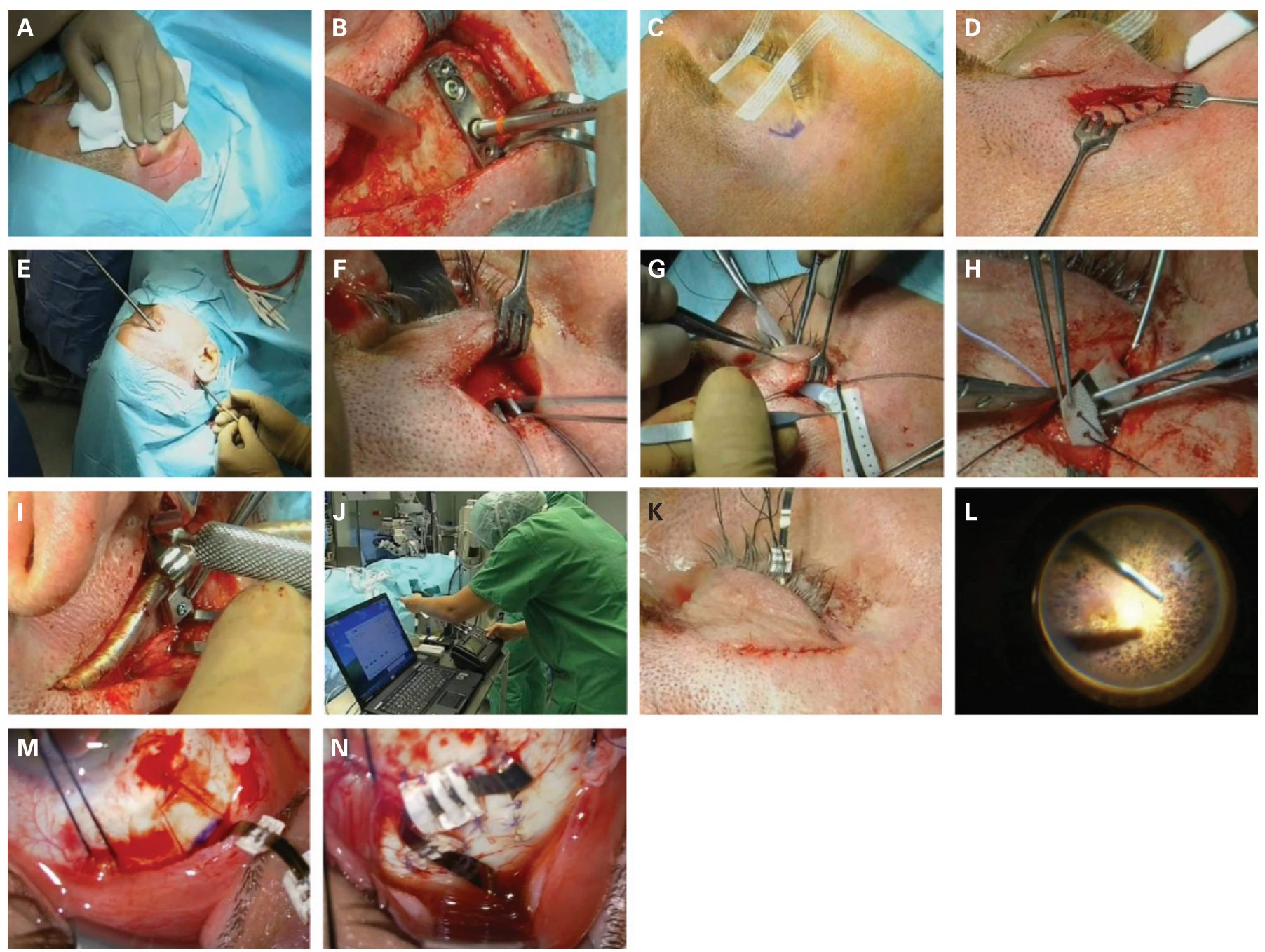

Figure 3 Photographs of the decisive surgical steps for implantation. (A) Localisation and marking of the retro-auricular cut line. (B) Exposition of the retro-auricular bone and fixation of the base of the fixation clamp with two osteosynthesis screws. (C) Localisation and marking of the elongated brow cut line. (D) Preparation of the lateral orbital region and marking of two holes (2 $\mathrm{mm}$ above and below the sutura zygomatico-frontalis) for fixation of the implant by sutures. (E) Introduction of the trocar in anterior-posterior direction beneath the temporal periosteum and insertion of the implant. (F) Blunt preparation from the subconjunctival space to the lateral orbital rim region. (G) Pulling of the polyimide foil with the subretinal parts protected in a silicone tube. (H) Fixation of the silicone patch by sutures to the lateral orbital rim. (I) Fixation of the top plate of the bone clamp to fixate the silicone cable in the retro-auricular region before skin perforation. (J) Electrical testing immediately before the intraocular surgery. (K) The implant lies on the sclera before the intraocular surgery; the brow cut has been closed; the two white patches for scleral fixation are visible. (L) Standard pars-planavitrectomy is performed. (M) Preparation of the scleral flap for choroidal penetration into the subretinal space in the upper lateral quadrant of the eye. (N) Fixation of the two fixation pads on the sclera.

haematomas had almost completely resolved, and patients were discharged.

All wounds healed properly, and no signs of infections or wound dehiscence were noticed. The skin penetration of the silicone cable proved to be tolerable and unproblematic over the whole examination period.

The retina was attached immediately postoperatively until explantation in all cases (fig 6 shows fundus photographs before, 1 week after, and 4 weeks after implantation). In the early postoperative days, some retinal oedema over the MPDA was present in optical coherence tomography (fig 7A). The amount of oedema decreased during the first postoperative week. Fluorescein angiography (fig 7B) showed capillaries in fine detail over the MPDA because the opaque MPDA totally blocked background fluorescence from the choroid. In the periphery (upper temporal part) of the MPDA capillary rarefication was noticeable, which could be due to either mechanical obstruction from the thickness of the soldering points of the MPDA in this region or pre-existent changes in RP retinas. $^{20}$

\section{Influence on ocular motility}

Before surgery, eye movements were normal in all patients, but various degrees of consecutive exotropia were noted in some patients. Directly after surgery (eg, in the patient in fig 5A), downgaze was restricted moderately, and a slight adduction and abduction deficit could be observed. Just before explantation, eye movements had improved (fig $5 \mathrm{~B}$ ).

\section{Explantation}

Prostheses were explanted after 4 weeks in patients 2, 3 and 4; and after 5 weeks in patients 5, 6 and 7 . The elongation of the 
Figure 4 Photographic aspects at various time points after implantation. (A) Frontal aspect 6 days after implantation. $(B, C)$ Frontal and retro-auricular aspects, respectively, 28 days after implantation. (D, E) Temporal and retro-auricular aspects, respectively, 2 weeks after explantation.
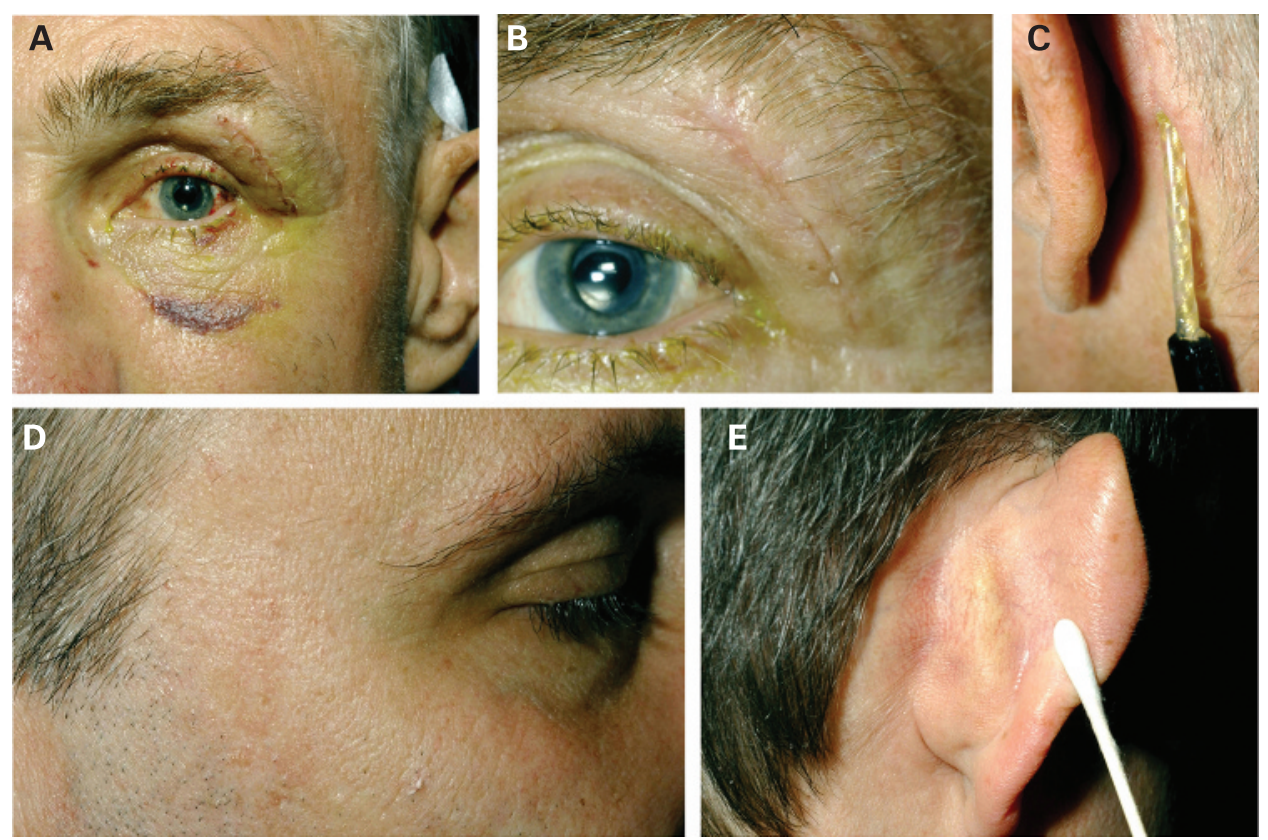

study period was permitted by the local ethics committee. Patient 1 refused to have the device explanted.

At explantation, the implant was surrounded by scar tissue, which, due to the inert nature of the polyimide, silicone and expanded polytetrafluoroethylene materials, was not connected to any of the implant's parts. Therefore, after careful dissection of this tissue using microscissors and removal of episcleral and bone sutures, the device could simply be pulled out as described above. Explantation was uneventful in all six cases, and patients could be discharged on the following day.

\section{DISCUSSION}

The study has proven that the surgical access developed for this study is feasible to implant a retinal prosthesis with extraocular parts for a period of at least 4 weeks. Surgery was successful in all cases and led to stable placement of the prosthesis over the entire study period. No postoperative complications were encountered. Patients felt astonishingly very little irritation with the surgical procedure and the presence of the device. Complaints were minimal and comparable with standard intraocular procedures. Patients did not feel restricted by the
Figure 5 Motility charts of one exemplary patient. (A) Six days after implantation. Downgaze was restricted moderately, and a very slight adduction and abduction deficit could be observed, accompanied by a mild tension during eye movements. The restriction was noticed by all patients, but no discomfort or subjective disturbance was reported. Various degrees of pre-existing consecutive exotropia were observed in this and all other patients. (B) Thirty days after implantation, immediately before explantation, eye movements were improved, and tension had disappeared.
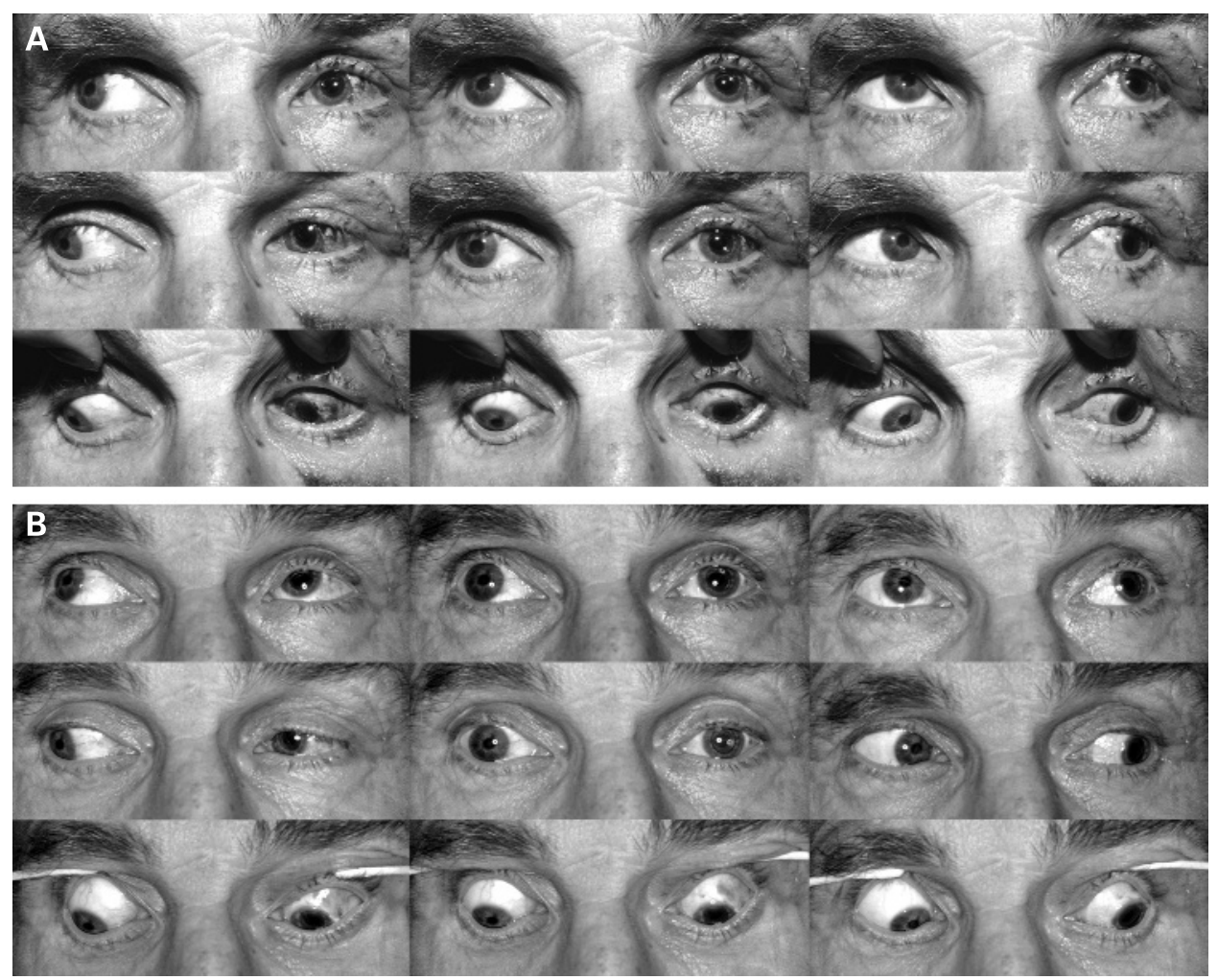

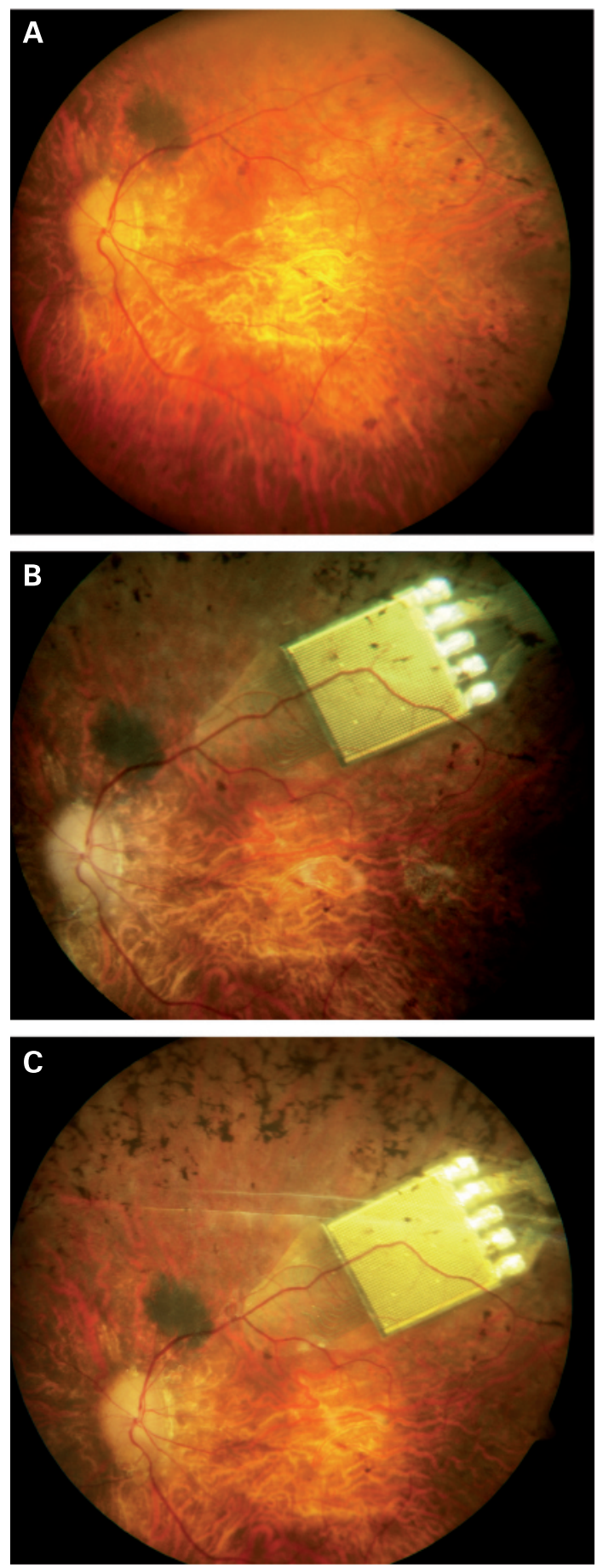

Figure 6 Fundus photography in one patient before implantation $(A)$, 1 week after (B) and 4 weeks after implantation (C). The tip of the subretinal polyimide foil which carries the electrode array for direct stimulation and the microphotodiode array is located parafoveally. The foil extends peripherally in the subretinal space to the transchoroidal penetration. The subretinal implant remained stable for the entire examination period. Retinas remained attached. Reflexes are from the silicone oil-retina interface.

device except for some eye movements, but in no cases was this described as disturbing. The transcutaneous connection proved to be uncomplicated and usable for repeated connection and disconnection of the stimulator. Patients were not required to take special care of the wound and cable; they were able to shower and perform all other tasks of daily life.
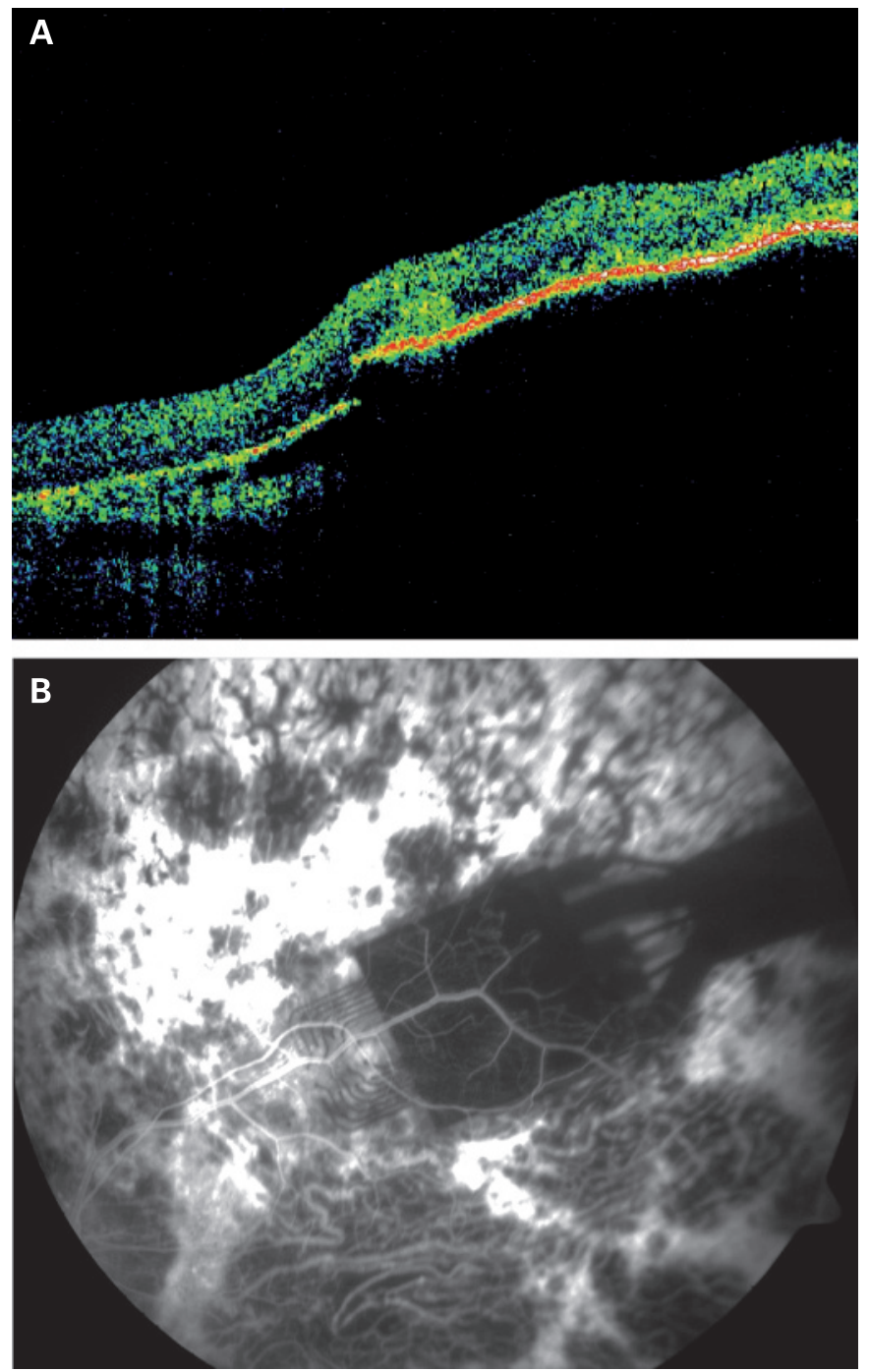

Figure 7 (A) Optical coherence tomography scan of the retina overlying the microphotodiode-array (MPDA) and the direct stimulation (DS) electrode-array 1 week postoperatively. The MPDA is the thick red line of high signal intensity on the right-hand side; the MPDA causes total extinction of the scan beam behind it. The DS array is the thinner red line on the left side. The retina over this part is normal in thickness and structure. The retina is attached over the entire subretinal implant and follows its curvature even at the site of the step from the thinner DS array to the thicker MPDA (20 $\mu \mathrm{m}$ and $100 \mu \mathrm{m}$, respectively). (B) Fluorescein angiography 1 week postoperatively. Retinal vasculature is visible in high detail over the MPDA since background fluorescence is totally blocked in this region. There is no dye leakage over the DS or over the MPDA area. However, capillary drop-out in the upper part over the MPDA is visible. This is the region where the thick soldering joints of the implant could represent a mechanical barrier leading to obstruction of vessels which are already impaired by the underlying disease.

In previous animal experiments, small dislocations of the subretinal parts of the implant have led to retinal tears and subsequently to retinal detachments. ${ }^{14}$ Therefore, techniques have been deployed by our group to ensure stable placement of the subretinal stimulation parts. ${ }^{14}$ For this purpose, multiple tension relief points were introduced along the course of the implant: flexible pads for fixation on the sclera, two sutures through holes in the bone of the lateral orbital rim and a metal clamp on the scull bone in the retro-auricular space for the 
silicone cable. Consequently, no retinal detachments or dislocations were observed in this human trial.

As mentioned, the greatest surgical challenge proved to be the intraoperative handling of the delicate structures of the implant. Since the implant could not be manufactured in separate connectible parts, the device had to be implanted in toto in the posterior-anterior direction requiring passage of the fine intraocular parts through the subdermal canal. At various steps during the procedure scissors, knives and coagulator forceps had to be used in closest proximity to the implant, which required great attention from the whole surgical team. However, the implant was not damaged in any case.

Humayun et al have reported on an epiretinal implant with a cable connection to a subdermal wireless receiver in the retroauricular region. ${ }^{21}$ According to the very brief description in the publication, a bone well was created to take up the cable from the lateral orbit to the retro-auricular space. There is no description of the procedure from the vitreous cavity to the orbit. From our experience, the creation of the bone well unnecessarily complicated the procedure and could be avoided in future procedures.

For this pilot trial, we have made use of an extracorporeal connector through a transcutaneous cable in order to be able to directly measure charge transfer and impedance of individual electrodes. This gave us valuable insight into the interface of electrodes and neural tissue from the subretinal space which has not been available from previous experiments, in particular not in semichronic experiments. Using a direct connection, we were also able to optimise the control settings for the MPDA, which will be used for the next implantations with a wirelessly controlled implant. It is clear that an extracorporeal connection does not qualify for permanent and routine use in patients. After the positive results of this study and the valuable information gathered for future implant generations, we feel, however, that this step was justified in this first human pilot trial with an active subretinal implant. This step of the surgery could, in addition, also be of potential interest under differing circumstances in experimental ophthalmic surgery.

To overcome this transcutaneous cable connection, the receiver of a wireless system has been either placed subcutaneously in the retro-auricular space, ${ }^{21}$ episclerally ${ }^{22}$ or even intraocularly as a modified intraocular lens in the capsular bag. ${ }^{23}$ Even when using up-to-date technology, a totally intraocularly implantable device is still large, and the trauma inferred for implantation is considerable, since it requires a large limbal opening and combined anterior and posterior segment surgery with a wired connection, for example from the capsular bag to the episcleral surface, ${ }^{23}$ or possibly even to the subretinal space. The easier surgical access and the additional room in the extraocular space give more freedom in choice for the receiver coils and control circuitry. In our opinion, therefore, a system where the receiver and control units of a wireless system are placed extraocularly has significant advantages. In particular, in regard to the unproblematic transchoroidal, trans-scleral penetration with a wired connection in our cases and in the literature 1416182124 a totally intraocularly implantable system does not seem essential. Several steps of the extraocular surgery presented in our study can contribute to implantation of such a prosthesis and also under other circumstances in ophthalmic research, for example when intraocular technical devices have to receive or transmit signals, such as intraocular pressure ${ }^{25}$ or temperature sensors, ${ }^{26}$ epi- or subretinal prostheses and also optic nerve head prosthesis. ${ }^{12}$
Acknowledgements: We gratefully acknowledge the help of our project partners to this work (Retina Implant AG, Reutlingen: WG Wrobel, R Rubow, H Sailer, A Harscher, $S$ Meine; Institute for Microelectronics, Stuttgart; Institute for Physical Electronics, Stuttgart: HG Graf, H Gruber; all Germany); Naturwissenschaftlich-medizinisches Institut Reutlingen: W Nisch, A Stett, H Hämmerle. G Fuchs was extremely helpful in surgery, as was A Bezirgiannidis in providing help in the operation theatre. R Hofer and U Greppmaier contributed fig 2. The German Ministry for Education and Research (BMBF, grant no 01KP0401) has generously supported our work.

Competing interests: According to the journal's guidelines, the authors declare the following: (1) the retinal implant was manufactured and delivered by Retina Implant AG (RIAG), Gerhard-Kindler-Straße 8, D-72770 Reutlingen, Germany; (2) RIAG covered the direct costs of the study; (3) HS, EZ and FG are shareholders of RIAG; none of them receives payment or is employed by RIAG.

Ethics approval: The ethics committee of the Medical Faculty of the University of Tübingen provided the approval.

Patient consent: Obtained.

\section{REFERENCES}

1. Rizzo JF III, Wyatt J, Humayun M, et al. Retinal prosthesis: An encouraging first decade with major challenges ahead. Ophthalmology 2001;108:13-14.

2. Zrenner E. Will retinal implants restore vision? Science 2002;295:1022-5.

3. Santos A, Humayun MS, de Juan EJ, et al. Preservation of the inner retina in retinitis pigmentosa. A morphometric analysis. Arch Ophthalmol 1997;115:511-15.

4. Stone JL, Barlow WE, Humayun MS, et al. Morphometric analysis of macular photoreceptors and ganglion cells in retinas with retinitis pigmentosa. Arch Ophthalmol 1992;110:1634-9.

5. Zrenner E, Miliczek K-D, Gabel V-P, et al. The development of subretinal microphotodiodes for replacement of degenerated photoreceptors [see comments] Ophthalmic Res 1997;29:269-80.

6. Zrenner E, Stett A, Weiss $\mathrm{S}$, et al. Can subretinal microphotodiodes successfully replace degenerated photoreceptors? Vision Res 1999;39:2555-67.

7. Schwahn HN, Gekeler F, Kohler K, et al. Studies on the feasibility of a subretinal visual prosthesis: data from Yucatan micropig and rabbit. Graefes Arch Clin Exp Ophthalmol 2001;239:961-7

8. Zrenner E. The subretinal implant: can microphotodiode arrays replace degenerated retinal photoreceptors to restore vision? Ophthalmologica 2002;216:8-20.

9. Stett A, Kohler K, Weiss S, et al. Electrical stimulation of degenerated retina of RCS rats by distally applied spatial voltage patterns. Invest Ophthalmol Vis Sci 1998;39:902S.

10. Stett A, Barth W, Haemmerle $\mathrm{H}$, et al. Network activity of the chicken retina electrically evoked by distally applied spatial voltage patterns. In: Elsner N, Wehner R, eds. Proceedings of the 26th Goettingen Neurobiology Conference. Stuttgart: Georg Thieme, 1998:437.

11. Stett A, Tepfenhart M, Kohler K, et al. Charge sensitivity of electrically stimulated chicken and RCS rat retinae. Invest Ophthalmol Vis Sci 1999;40:734S

12. Stett A, Barth W, Weiss $S$, et al. Electrical multisite stimulation of the isolated chicken retina. Vision Res 2000;40:1785-95.

13. Gekeler F, Kobuch K, Schwahn HN, et al. Subretinal electrical stimulation of the rabbit retina with acutely implanted electrode arrays. Graefes Arch Clin Exp Ophthalmol 2004;242:587-96.

14. Gekeler F, Szurman P, Grisanti S, et al. Compound subretinal prostheses with extraocular parts designed for human trials: successful long-term implantation in pigs. Graefes Arch Clin Exp Ophthalmol 2007;245:230-41.

15. Sachs HG, Gabel VP. Retinal replacement-the development of microelectronic retinal prostheses - experience with subretinal implants and new aspects. Graefes Arch Clin Exp Ophthalmol 2004;242:717-23.

16. Sachs HG, Schanze T, Brunner U, et al. Transscleral implantation and neurophysiological testing of subretinal polyimide film electrodes in the domestic pig in visual prosthesis development. J Neural Eng 2005;2:57-64S.

17. Sachs HG, Gekeler F, Schwahn H, et al. Implantation of stimulation electrodes in the subretinal space to demonstrate cortical responses in Yucatan minipig in the course of visual prosthesis development. Eur J Ophthalmol 2005;15:493-9.

18. Sachs HG, Schanze T, Wilms M, et al. Subretinal implantation and testing of polyimide film electrodes in cats. Graefes Arch Clin Exp Ophthalmol 2005;243:464-8.

19. Gekeler F, Messias A, Ottinger M, et al. Phosphenes electrically evoked with DTL electrodes: a study in patients with retinitis pigmentosa, glaucoma, and homonymous visual field loss and normal subjects. Invest Ophthalmol Vis Sci 2006;47:4966-74.

20. Gekeler F, Sachs H, Szurman P, et al. Fluorescein angiographic findings in blind patients treated by an active subretinal implant. Invest Ophthalmol Vis Sci 2007; 48:2567.

21. Humayun MS, Weiland JD, Fujii GY, et al. Visual perception in a blind subject with a chronic microelectronic retinal prosthesis. Vision Res 2003:43:2573-81.

22. Rizzo JF III, Chen J, Shire D, et al. Implantation of a wirelessly powered retinal prosthesis using an ab externo surgical technique. Invest Ophthalmol Vis Sci 2008:49:3027. 
23. Alteheld N, Roessler G, Walter P. Towards the bionic eye-the retina implant: surgical, opthalmological and histopathological perspectives. Acta Neurochir Suppl 2007; 97:487-93

24. Sachs HG, Bartz-Schmidt U, Brunner U, et al. Subretinal chronic active visual prostheses in blind patients: the transchoroidal surgical procedure. Invest Ophthalmol Vis Sci 2006; 47:3207.
25. Walter P, Schnakenberg U, vom Bögel G, et al. Development of a completely encapsulated intraocular pressure sensor. Ophthalmic Res 2000;32:278-84

26. Sailer H, Shinoda K, Blatsios G, et al. Investigation of thermal effects of infrared lasers on the rabbit retina: a study in the course of the development of an active subretinal prosthesis. Graefes Arch Clin Exp Ophthalmol 2007;245:1169-78.

\section{Education}

\author{
ANSWERS \\ From questions on page 1311
}

\section{How can an astigmatically neutral wound be achieved in DSAEK?}

The creation of a self-sealing scleral tunnel in DSAEK using an "anti-smile" incision, as described in this surgical technique, does not require application of sutures, making the wound astigmatically neutral.

\section{How can the surgeon ensure an easy and atraumatic insertion of the donor lenticule into the recipient's AC?}

The use of the Busin glide eliminates folding of the lenticule. The graft can be easily pulled from the glide inside the AC of the host using forceps. Also, the leading edge of the donor lenticule can be stained with Gentian Violet to facilitate its visualisation while attempting to grasp it with crocodile forceps. Furthermore, reducing the radial distance between the Busin glide and forceps to $110-120^{\circ}$ avoids obstruction to the movement of the surgeon's hand caused by the lower orbital rim and helps improve manipulation of forceps.

3. What are the advantages of creation of fenestration wounds, prior to insertion of donor lenticule?

Creation of stab incisions at the end of surgery can cause a large amount of pressure on the donor lenticule and may displace it. Creation of these stab incisions before the insertion of the graft can eliminate the occurrence of such a complication.

\section{DISCUSSION}

DSAEK is a preferred technique of corneal transplantation for corneal endothelial dysfunction. We made certain modifications in the standard surgical technique in an endeavour to minimise complications and make the surgery more reproducible. ${ }^{4}$

\section{Surgical wound}

One of the modifications is the creation of a scleral tunnel using an "anti-smile" incision. This type of surgical wound is selfsealing and does not require application of sutures, therefore making it astigmatically neutral

\section{Marking the edge of donor lenticule}

We observed that in eyes with postcataract surgery superior corneal scar, superior corneal pannus or preoperative corneal oedema, the translucent margin of donor lenticule was not clearly visible. Staining the leading edge of the donor lenticule with Gentian Violet made its visualisation easier inside the clear corneal part of the tunnel.

\section{Distance between main wound and side port for forceps}

The recommended radial distance between glide and forceps is $180^{\circ}$. We observed that reducing this distance facilitated easy pulling of the donor lenticule inside the recipient's AC.

\section{Use of the Busin glide}

Prevention of endothelial cell loss has been a key issue associated with DSAEK due to folding of donor lenticule. ${ }^{5}$ The use of the Busin glide eliminates folding of the lenticule and facilitates pulling of the graft from the glide inside the AC of the host using forceps.

\section{Creation of stained fenestration wounds for drainage before the insertion of donor lenticule}

Drainage of interface fluid using the "fenestration technique" has significantly reduced the incidence of postoperative graft dislocations. ${ }^{5-7}$ We created four full-thickness stab incisions prior to insertion of the donor lenticule to avoid putting pressure on the donor lenticule. We also marked these with Gentian Violet. Staining of stab wounds can help in identifying these easily in case the fluid needs to be drained during the early postoperative period. [8]

We believe that these small modifications, particularly the use of the Busin glide, are a useful addition to the present technique and would help alleviate the learning curve associated with DSAEK.

\section{Br J Ophthalmol 2008;92:1368. doi:10.1136/bjo.2008.139121a}

\section{REFERENCES}

1. Price FW Jr, Price MO. Descemet's stripping with endothelial keratoplasty in 50 eyes: a refractive neutral corneal transplant. J Refract Surg 2005;21:339-45.

2. Terry MA. Endothelial keratoplasty: history, current state, and future directions Cornea 2006:25:873-8.

3. Price MO, Price FW Jr. Descemet's stripping with endothelial keratoplasty: comparative outcomes with microkeratome-dissected and manually dissected donor tissue. Ophthalmology 2006;113:1936-42.

4. Vajpayee RB, Agarwal T, Jhanji V, et al. Modification in Descemet-stripping automated endothelial keratoplasty: "Hitch suture" technique. Cornea 2006:25:1060-2.

5. Gorovoy MS. Descemet-stripping automated endothelial keratoplasty. Cornea 2006;25:886-9.

6. Terry MA, Hoar K, Wall J, et al. Histology of dislocations in endothelial keratoplasty (DSEK and DLEK): a laboratory-based, surgical solution to dislocation in 100 consecutive DSEK cases. Cornea 2006;25:926-32.

7. Price FW Jr, Price MO. Descemet's stripping with endothelial keratoplasty in 200 eyes: Early challenges and techniques to enhance donor adherence. J Cataract Refract Surg 2006:32:411-18

8. Srinivasan S, Rootman DS. Slit-lamp technique of draining interface fluid following Descemet's stripping endothelial keratoplasty. Br J Ophthalmol 2007;91:1202-5. 